\title{
Autarke multiparametrische Monitoring-Systeme für die Biogasproduktion zur Optimierung ihrer Leistungsparameter
}

\author{
Michael Tietze ${ }^{1}$ \\ Katja Hahne ${ }^{2}$, Susann Götze ${ }^{2}$, Kai Ostermann², Gerhard Rödel2 \\ Lisa Birkeneder ${ }^{3}$, Jens Zosel ${ }^{3}$, Juliane Posseckardt ${ }^{3}$,Christine Schirmer ${ }^{3}$, Michael Mertig $^{3}$ \\ Steffen Howitz ${ }^{4}$ \\ ${ }^{1}$ GICON - Großmann Ingenieur Consult GmbH, 01219 Dresden/Deutschland \\ ${ }^{2}$ Institut für Genetik, Technische Universität Dresden; 01062 Dresden/Deutschland \\ ${ }^{3}$ Kurt-Schwabe-Institut für Mess- und Sensortechnik e.V. Meinsberg, 04736 Waldheim/Deutschland \\ ${ }^{4} \mathrm{GeSiM}$ - Gesellschaft für Silizium-Mikrosysteme mbH, 01454 Radeberg/Deutschland \\ m.tietze@gicon.de
}

\begin{abstract}
Zusammenfassung
Um biotechnologische Prozesse effizient und sicher betreiben zu können, bedarf es einer leistungsfähigen Mess- und Analysentechnik. Ziel des Wachstumskerns BioSAM - TP Biogas - ist es, ein inline arbeitendes Mess- und Auswertesystem für die Zustandsbeschreibung von biotechnologischen Prozessen zu entwickeln und unter praxisnahen Bedingungen zu testen. Als Referenzanwendung ist dabei das Monitoring von Prozessbedingungen bei der Biogaserzeugung vorgesehen. Mit dem System sollen wichtige biochemische Prozessindikatoren spezifisch erfasst werden. Dabei stehen zunächst in der Prozessflüssigkeit gelöste Spurenelemente sowie Essigsäure als wesentliche Parameter für die Beurteilung der Prozessstabilität und Leistungsfähigkeit von Biogasprozessen im Fokus.

Als hoch sensitive Schnittstelle des zu entwickelnden Messsystems zu den biotechnologischen Prozessen dienen immobilisierte Zellen, mit denen die Konzentrationen der gewünschten Komponenten erfasst und über neu entwickelte Transducer (Sensorwandler) an ein gemeinsames System der Plattform zur Signalverarbeitung übergeben werden.

Arbeitsschwerpunkte des Projektes bilden die Herstellung von Zellen mit sensorischen Eigenschaften zur Detektion von Spurenelementen und Zwischenprodukten biotechnologischer Prozesse, deren Charakterisierung und Optimierung in Hinblick auf Sensitivität, Selektivität und Stabilität sowie Immobilisierung, Versorgung, Zustandsmonitoring und Signalauswertung dieser Zellen in speziell entwickelten Sensorkompartimenten. Darüber hinaus erfolgt die Entwicklung, Herstellung und Erprobung von mikrofluidischen und optischen Komponenten zur Ansteuerung der Sensorkompartimente und zur Signalauswertung, Zellbeurteilung und Referenzierung der Messungen und eine hardwaretechnische Einbindung der Sensoreinheiten in ein Multiparameter-Messsystem.

Um eine möglichst praxisnahe Entwicklung zu gewährleisten, erfolgen mit den Sensoren bzw. dem Messsystem Tests an labor-, halbtechnischen und großtechnischen Anlagen.
\end{abstract}

Keywords: Nachweis von Essigsäure,Hefe-Ganzzellsensoren,Mikrofluidik

\section{Einleitung}

Ein Leitparameter für die Zustandsbeschreibung des Biogasprozesses stellt das Zwischenprodukt Essigsäure dar. Eine Akkumulation von Essigsäure ist ein Indikator für eine Prozessstörung, ausgelöst durch ein Ungleichgewicht aus Säureproduzenten und konsumenten [1]. Eine kontinuierliche Überwachung der Essigsäurekonzentration in
Biogasanlagen ermöglicht es somit, Prozessstörungen frühzeitig zu erkennen und kann für die Optimierung sowie für die Steuerung des Biogasprozesses genutzt werden.

Spurenelemente wie Kupfer- und Eisenionen sind ebenfalls wichtige Parameter für die Biogasproduktion, da sie von den Mikroorganismen als essentielle Co-Faktoren benötigt werden. Die Bestimmung dieser Parameter ist derzeit mit hohen Kosten 
verbunden und oft nur zeitversetzt möglich. Darüber hinaus liefert die Analyse keine Aussagen, inwieweit die untersuchten Analyten in dem hoch komplexen System Biogasreaktor in einer für die Mikroorganismen verfügbaren Form vorliegen. Um dies zu ermöglichen, stellt die Sensorik von Essigsäure sowie der genannten Spurenelemente durch HefeGanzzellsensoren einen Schwerpunkt im Projekt Biogas dar.

Nach der Definition der IUPAC ${ }^{1}$ ist ein Biosensor ein in sich geschlossenes integriertes System, das in der Lage ist, quantitative oder halb-quantitative analytische Informationen zu liefern. Das biologische Erkennungselement (biochemischer Rezeptor) steht dabei in direktem räumlichen Kontakt mit dem Transducer (Signalumwandler), der als Reaktion auf die Veränderung der Umwelt ein messbares Signal produziert. Biosensoren zeichnen sich durch hohe Spezifität und Sensitivität aus. Ein Vorteil von Ganzzellsensoren ist, dass sie Informationen über die Bioverfügbarkeit des Analyten liefern [2,3]. Für die Generierung von Ganzzellsensoren eignen sich Hefen in besonderem $\mathrm{Maße}$, da sie u.a. physisch sehr robust sind, nur geringe Wachstumsanforderungen stellen und genetisch einfach zu manipulieren sind [4].

\section{Generierung von Ganzzellsensoren}

Verschiedenste Moleküle/Analyten werden von Hefezellen wahrgenommen und führen direkt oder indirekt zur Aktivierung spezifischer Transkriptionsfaktoren (TF), die sich an responsive Elemente im 5'-regulatorischen Bereich (Promotor) von Genen anlagern. Durch die Bindung des TF wird die Transkription des entsprechenden Gens verstärkt (Abb. 1A). Dies wurde für die Generierung von Ganzzellsensoren genutzt: Eine Promotorsequenz, an die ein Essigsäurespezifischer TF binden kann, wurde mit dem Gen für ein fluoreszierendes Protein gekoppelt. Dieses Reportergenkonstrukt wurde in Hefezellen transformiert und somit entsprechende Ganzzellsensoren generiert (Abb. 1B).

\footnotetext{
1 International Union of Pure and Applied Chemistry
}

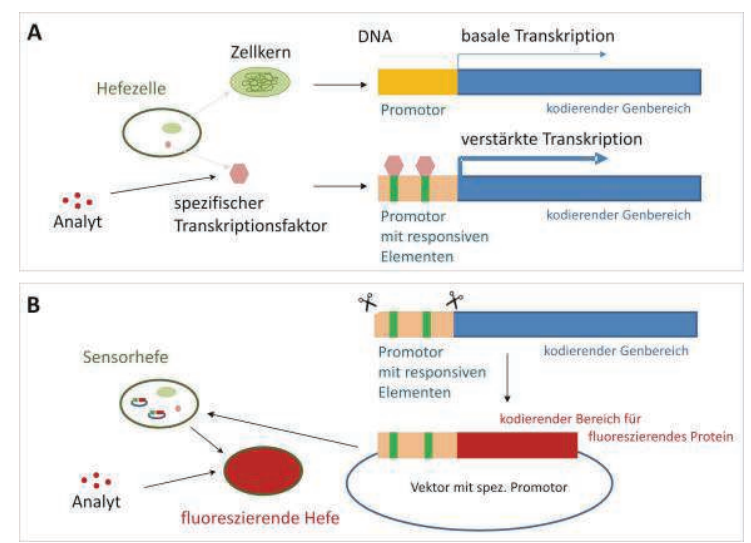

Abb. 1: Allgemeine Strategie zur Generierung von Sensorhefen. A stellt die verstärkte Transkription aufgrund der Bindung eines spezifischen Transkriptionsfaktors an den Promotorbereich eines Genes schematisch dar. B zeigt das allgemeine Vorgehen zur Generierung von Ganzzellsensoren auf Plasmidbasis.

\section{Detektion von Essigsäure}

Die Ansprechbarkeit der Ganzzellsensoren durch Essigsäure wurde initial durch Fluoreszenzmessungen im Plattenreader getestet. Eine prinzipielle Eignung der Zellen für den Nachweis von Essigsäure konnte gezeigt werden (K. Hahne, unveröffentlicht).

\section{Langzeitstabilität}

Hefen bilden als langzeitstabile Dauerform Sporen. Diese sollen zur Lagerung der Ganzzellsensoren genutzt werden. Dazu wurde das Reportergenkonstrukt in das Genom der Hefe haploider Zellen integriert, diploide Zellen erzeugt und diese zur Sporulation gebracht. Die Sporen wurden unter verschiedenen Bedingungen gelagert. $\mathrm{Zu}$ festgelegten Zeitpunkten erfolgten eine Reaktivierung vegetativer Hefezellen aus den Sporen und die Analyse der durch Essigsäure ausgelösten Fluoreszenz. Es konnte gezeigt werden, dass die Lagerung bis zu einem Zeitraum von sechs Monaten die Reaktionsfähigkeit des Ganzzellsensors nicht beeinflusst (K. Hahne, unveröffentlicht).

\section{Einhausung und mikrofluidischer Sensoraufbau}

Der Einsatz der generierten Essigsäuresensitiven Hefezellen zur Detektion der Essigsäurekonzentration in Biogasanlagen erfordert einen mikrofluidischen Sensoraufbau, welcher folgende Anforderungen erfüllen soll: (i) Einhausung der gentechnisch modifizierten 
Hefezellen (ii) Zufuhr von Nährstoffen und Analyt zu den Hefezellen und (iii) Auslese des Fluoreszenzsignales. Der im Rahmen des Wachstumskernes BioSAM entwickelte Sensoraufbau basiert auf dem MicCell $^{\text {TM }}$ System [5] der Firma GeSiM. Er ist so aufgebaut, dass die Hefezellkammer durch eine Membran von dem Versorgungskanal getrennt ist, welche die Hefezellen nicht passieren können. Es konnte gezeigt werden, dass die Hefezellen den sensorischen Aufbau nicht verlassen können. Weiterhin konnte sowohl experimentell als auch per Simulation nachgewiesen werden, dass die Hefezellen über den Versorgungskanal ausreichend mit Nährstoffen versorgt werden können. Diese Ergebnisse werden im Tagungsbeitrag von Schirmer/Günther et al. am Beispiel der Diclofenac-Detektion dargestellt.

Die Entwicklung des Anteils fluoreszierender Zellen und die Fluoreszenzintensität pro Zelle bei Inkubation der Hefezellkulturen mit Essigsäure und weiteren flüchtigen Fettsäuren wurden mit dem Durchflusszytometer untersucht. Diese Daten wurden zur Interpretation der Fluoreszenzmessungen im mikrofluidischen Sensoraufbau herangezogen. Es konnte eine konzentrationsabhängige Entwicklung der Fluoreszenz auf Essigsäurekonzentrationen zwischen 1 und $10 \mathrm{mM}$ gezeigt werden (L. Birkeneder, unveröffentlicht).

\section{Vom Laboraufbau zum Anwender}

Die Detektion der Fluoreszenz in dem oben beschriebenen mikrofluidischen Sensoraufbau erfolgt bisher über die Aufnahme von Mikroskopbildern und die Auswertung der mittleren Grauwerte. Da diese Detektion in der Praxis nicht anwendbar ist, wurden im Teilprojekt HIGS verschiedene alternative Detektionseinheiten auf der Basis eines Spektrometers bzw. von Photodioden aufgebaut. Diese werden ausführlich im Tagungsbeitrag von M. Schröder et al. vorgestellt:

Weiterhin wurde von der Firma GeSiM eine Folien-Flowzelle entwickelt, welche als Einweg-Alternative für die oben beschriebene PDMS-Flowzelle dienen soll. Auch hier verhindert eine semipermeable Membran, dass die Zellen den Sensor verlassen können (S. Howitz, unveröffentlicht).

\section{Referenzmessungen}

Die Referenzierung der neu entwickelten Messmöglichkeiten für Essigsäure und Spurenelemente in Biogasmedien erfordert die präzise Bestimmung dieser Analyte mit standardisierten Methoden. Im Fall der
Spurenelemente steht bereits eine Reihe von Labormethoden zur Verfügung [6], mit denen diese zuverlässig in entnommenen Gärsubstraten quantitativ bestimmt werden können. Im Fall der Essigsäure haben Ringversuche gezeigt, dass hier die Probenahme und anschließende Bestimmung im Labor Fehlerquellen beinhaltet, die zu so hohen Abweichungen führen können, dass diese Methode nur sehr eingeschränkt als zuverlässige Referenzierung in Frage kommt [7]. Um hier einen verbesserten Ansatz zu verfolgen, wurde das im Beitrag von Zosel et al. in diesem Tagungsband genauer beschriebene automatische Messsystem entwickelt, mit dem eine präzise OnlineMessung flüchtiger Fettsäuren in Biogaskondensaten nach chromatographischer Trennung möglich ist. Eine Beispielmessung an einem Kondensat aus einer Technikumsanlage mit diesem Messsystem ist in Abb. 2 dargestellt.

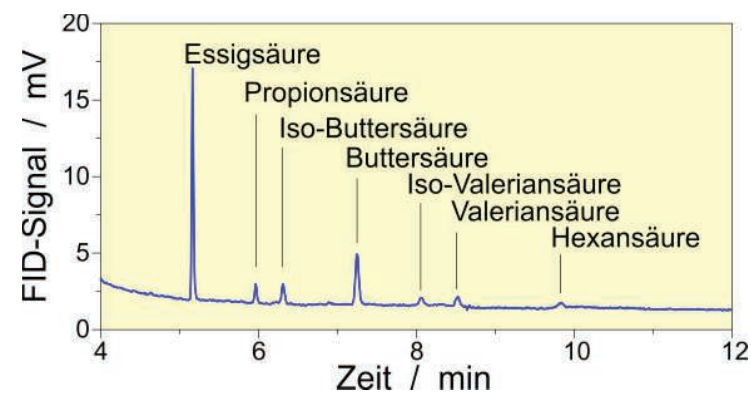

Abb. 2: Chromatogramm einer Kondensatprobe aus einem realen BiogasProzess mit intensivierter Fütterung, Einspritzvolumen $20 \mu \mathrm{L}$.

Ein Vergleich der Referenzmessungen mit denen der Ganzzellsensorik zeigt gute Erfolgschancen auf, dass die Essigsäurekonzentration in Kondensatproben einer Biogasanlage durch das neu entwickelte HefeGanzzellsensorsystem mit hoher Präzision bestimmt werden kann.

\section{Danksagung}

Die Autoren danken dem Bundesministerium für Bildung und Forschung für die Förderung des Verbundprojektes BIOGAS im Wachstumskern BioSAM (Förderkennzeichen 03WKCLO4D).

\section{Literaturnachweis}

[1] M.S. Switzenbaum, E. Giraldo-Gomez, R.F. Hickey, Enzyme and Microbial Technology 12, 722-730 (1990); doi: 10.1016/01410229(90)90142-D 
[2] S. Daunert, G. Barrett, J.S. Feliciano, R.S Shetty, S. Shrestha, W. Smith-Spencer, Chemical Reviews 100, 2705-2738 (2000); doi: 10.1021/cr990115p

[3] D.R. Thévenot, K. Toth, R.A. Durst, G.S. Wilson, Biosensors and Bioelectronics 16, 121-131 (2001); doi: 10.1016/S09565663(01)00115-4

[4] R.M. Walmsley, P. Keenan, Biotechnology and Bioprocess Engineering 5, 387-394 (2000); doi: 10.1007/BF02931936]

[5] F.U. Gast, P.S. Dittrich, P. Schwille, M. Weigel, M. Mertig, J. Opitz, U. Queitsch, S. Diez, B. Lincoln, F. Wottawah, S. Schinkinger, J. Guck, J. Käs, J. Smolinski, K. Salchert, C. Werner, C. Duschl, M.S. Jäger, K. Uhlig, P. Geggier, S.

Howitz, Microfluidics and Nanofluidics 2, 21-36 (2006); doi: 10.1007/s10404-005-0047-6

[6] M. Ortner, BIOGAS Journal 2, 82-85 (2012)

[7] G. Henkelmann, K. Meyer zu Köcker, A. Gronauer, M. Effenberger, H. Heuwinkel, M. Lebuhn, K. Koch, Laboranalytik, BayernBiogasforum III, 1-10 (2012) 